Bull. Austral. Math. Soc.

VOL. 67 (2003) [377-382]

\title{
WEIGHTED DISCREPANCY OF FAURE-NIEDERREITER NETS FOR A CERTAIN SEQUENCE OF WEIGHTS
}

\section{FRIEDRICH PILLICHSHAMMER}

In this note we give a bound for the weighted star discrepancy of Faure-Niederreiter $(0, m, s)$-nets in prime-power base $b \geqslant s-1$ for the special sequence of weights given by $\gamma_{i}=1 / i^{2}, i \geqslant 1$.

Quite recently Sloan and Woźniakowski [4] introduced a new notion of discrepancy, the so called weighted star discrepancy. To introduce this notion we need a sequence $\gamma=\left(\gamma_{1}, \gamma_{2}, \ldots\right)$ of weights with $1=\gamma_{1} \geqslant \gamma_{2} \geqslant \ldots \geqslant \gamma_{s} \geqslant \ldots>0$.

First we need some notation: let $D$ denote the index set $D=\{1,2, \ldots, s\}$. For $\mathfrak{u} \subseteq D$ let $\gamma_{\mathfrak{u}}=\prod_{j \in \mathfrak{u}} \gamma_{j}, \gamma_{\emptyset}=1,|\mathfrak{u}|$ the cardinality of $u$ and for a vector $\mathbf{x}$ in $I^{s}=[0,1)^{s}$ let $\mathbf{x}_{\mathbf{u}}$ denote the vector from $I^{|u|}=[0,1)^{|u|}$ containing the components of $\mathbf{x}$ whose indices are in $u$. Further let $\left(\mathbf{x}_{\mathbf{u}}, 1\right)$ be the vector $\mathbf{x}$ from $I^{s}$, with all components whose indices are not in $u$ replaced by 1 .

Now we can give the definition of weighted star discrepancy.

Definition 1: For any $N$ points $t_{1}, \ldots, t_{N}$ in the $s$-dimensional unit cube $I^{s}$ and any $\mathbf{x}=\left(x_{1}, \ldots, x_{s}\right)$ in $I^{s}$ let

$$
\operatorname{disc}(\mathbf{x})=\frac{\left|\left\{i: \mathrm{t}_{i} \in[\mathbf{0}, \mathbf{x})\right\}\right|}{N}-x_{1} \ldots x_{s} .
$$

Then the weighted star discrepancy (with respect to the sequence $\gamma$ ) is given by

$$
D_{N, \gamma}^{*}=\sup _{\mathbf{x} \in I^{*}} \max _{\substack{u \subseteq D \\ u \neq \emptyset}} \gamma_{\mathfrak{u}}^{1 / 2}\left|\operatorname{disc}\left(\left(\mathbf{x}_{\mathbf{u}}, 1\right)\right)\right| .
$$

The weighted star discrepancy is related to the worst case error of multivariate integration in weighted Sobolev spaces of functions on $[0,1]^{s}$. For more information in this direction we refer to [4]. In [2] Larcher, Pillichshammer and Scheicher gave first good and effectively useful bounds for the weighted star discrepancy for special kinds of $(t, m, s)$-nets, where by "effectively useful" they meant that the obtained bounds can be calculated also for high dimensions in reasonable time.

Received 18th July, 2002

Copyright Clearance Centre, Inc. Serial-fee code: 0004-9727/03 \$A2.00+0.00. 
In this note we consider a very special sequence of weights, namely the sequence $\gamma$ $=\left(\gamma_{1}, \gamma_{2}, \ldots\right)$ given by $\gamma_{i}=1 / i^{2}$, for $i \geqslant 1$. As H. Woźniakowski (private communication) pointed out, this special sequence of weights may be relevant for finance problems since the eigenvalues of the covariance function for Wiener measure are proportional to this weights (see [1] ). So this motivates a more detailed study of weighted star discrepancy with respect to this special sequence of weights.

Here we shall prove a bound for the weighted star discrepancy with respect to the weights $\gamma_{i}=1 / i^{2}$, for $i \geqslant 1$, for Faure-Niederreiter point sets, which are most frequently used today in quasi-Monte Carlo applications. These point sets which are based on FaureNiederreiter sequences in dimension $s-1$ (see for example [3, Remark 4.52]) are $(0, m, s)$ nets in a prime-power base $b \geqslant s-1$. More detailed, a Faure-Niederreiter $(0, m, s)$-net in a prime-power base $b \geqslant s-1$ is obtained by setting $\mathbf{x}_{n}=\left(n / b^{m}, \mathbf{y}_{n}\right) ; n=0, \ldots, b^{m}-1$ where $\mathbf{y}_{n}$ is the $n$-th element of a Faure-Niederreiter sequence in dimension $s-1$ and prime-power base $b \geqslant s-1$.

We have

THEOREM 1. Let the sequence $\gamma=\left(\gamma_{1}, \gamma_{2}, \ldots\right)$ of weights be given by $\gamma_{i}=1 / i^{2}$, $i \geqslant 1$. Then we have: for any Faure-Niederreiter $(0, m, s)$-net in prime-power base $b \geqslant s-1$ the weighted star discrepancy $D_{N, \gamma}^{*}$ is bounded by

$$
D_{N, \gamma}^{*} \leqslant\left(\left\lfloor\begin{array}{c}
\frac{m}{2} \\
\left.\frac{m}{2}\right\rfloor
\end{array}\right) \frac{1}{b^{m}} \frac{1}{p !}\left(1+\left\lfloor\frac{b}{2}\right\rfloor\right)^{p-1}\right.
$$

where $N=b^{m}$ and $p=\min \{m+1,\lfloor b / 2\rfloor, s\}$.

A detailed discussion of the bound from Theorem 1 will be given at the end of this note.

Proof: From [2, Example 2.6] we know that for any Faure-Niederreiter $(0, m, s)$-net in base $b$ and for the special choice of weights the inequality

$$
N D_{N, r}^{*} \leqslant \max _{r=1, \ldots, s} \frac{1}{r !} \sum_{i=0}^{r-1}\left(\begin{array}{c}
r-1 \\
i
\end{array}\right)\left(\begin{array}{c}
m \\
i
\end{array}\right)\left\lfloor\frac{b}{2}\right\rfloor^{i}
$$

holds. Thus, letting

$$
h(r):=\frac{1}{r !} \sum_{i=0}^{r-1}\left(\begin{array}{c}
r-1 \\
i
\end{array}\right)\left(\begin{array}{c}
m \\
i
\end{array}\right)\left\lfloor\frac{b}{2}\right\rfloor^{i}
$$

we have to estimate $\max _{r=1, \ldots, s} h(r)$. 
Let be $r \geqslant 1$. Then we have

$$
\begin{aligned}
h(r)- & h(r+1) \\
& =\frac{1}{r !} \sum_{i=0}^{r-1}\left(\begin{array}{c}
r-1 \\
i
\end{array}\right)\left(\begin{array}{c}
m \\
i
\end{array}\right)\left\lfloor\frac{b}{2}\right\rfloor^{i}-\frac{1}{(r+1) !} \sum_{i=0}^{r}\left(\begin{array}{c}
r \\
i
\end{array}\right)\left(\begin{array}{c}
m \\
i
\end{array}\right)\left\lfloor\frac{b}{2}\right\rfloor^{i} \\
& =\frac{1}{r !} \sum_{i=0}^{r-1}\left[\left(\begin{array}{c}
r-1 \\
i
\end{array}\right)-\frac{1}{r+1}\left(\begin{array}{c}
r \\
i
\end{array}\right)\right]\left(\begin{array}{c}
m \\
i
\end{array}\right)\left\lfloor\frac{b}{2}\right\rfloor^{i}-\frac{1}{(r+1) !}\left(\begin{array}{c}
m \\
r
\end{array}\right)\left\lfloor\frac{b}{2}\right\rfloor^{r} \\
& =\frac{1}{(r+1) !} \sum_{i=0}^{r-1}\left[r\left(\begin{array}{c}
r-1 \\
i
\end{array}\right)-\left(\begin{array}{c}
r-1 \\
i-1
\end{array}\right)\right]\left(\begin{array}{c}
m \\
i
\end{array}\right)\left\lfloor\frac{b}{2}\right\rfloor^{i}-\frac{1}{(r+1) !}\left(\begin{array}{c}
m \\
r
\end{array}\right)\left\lfloor\frac{b}{2}\right\rfloor^{r} .
\end{aligned}
$$

Hence we have $h(r) \leqslant h(r+1)$ if and only if

$$
\sum_{i=0}^{r-1}\left[r\left(\begin{array}{c}
r-1 \\
i
\end{array}\right)-\left(\begin{array}{c}
r-1 \\
i-1
\end{array}\right)\right]\left(\begin{array}{c}
m \\
i
\end{array}\right)\left\lfloor\frac{b}{2}\right\rfloor^{i} \leqslant\left(\begin{array}{c}
m \\
r
\end{array}\right)\left\lfloor\frac{b}{2}\right\rfloor^{r}
$$

and $h(r) \geqslant h(r+1)$ if and only if

$$
\sum_{i=0}^{r-1}\left[r\left(\begin{array}{c}
r-1 \\
i
\end{array}\right)-\left(\begin{array}{c}
r-1 \\
i-1
\end{array}\right)\right]\left(\begin{array}{c}
m \\
i
\end{array}\right)\left[\frac{b}{2}\right\rfloor^{i} \geqslant\left(\begin{array}{c}
m \\
r
\end{array}\right)\left\lfloor\frac{b}{2}\right\rfloor^{r}
$$

Assume $r \geqslant m+1$. Then we have $\left(\begin{array}{c}m \\ r\end{array}\right)\lfloor b / 2\rfloor^{r}=0$. Since $r\left(\begin{array}{c}r-1 \\ i\end{array}\right)-\left(\begin{array}{c}r-1 \\ i-1\end{array}\right) \geqslant 1$ for $0 \leqslant i \leqslant r-1$ this implies

$$
h(r) \geqslant h(r+1)
$$

and hence we have (if $m+1 \leqslant s$ )

$$
\max _{r=1, \ldots, s} h(r)=\max _{r=1, \ldots, m+1} h(r) .
$$

So we estimate

$$
\max _{r=1, \ldots, m+1} h(r)
$$

Let $1 \leqslant r \leqslant m+1$. Then we have

$$
\begin{aligned}
h(r) & =\frac{1}{r !} \sum_{i=0}^{r-1}\left(\begin{array}{c}
r-1 \\
i
\end{array}\right)\left(\begin{array}{c}
m \\
i
\end{array}\right)\left\lfloor\frac{b}{2}\right\rfloor^{i} \\
& \leqslant \max _{i=0, \ldots, m}\left(\begin{array}{c}
m \\
i
\end{array}\right) \frac{1}{r !}\left(1+\left\lfloor\frac{b}{2}\right\rfloor\right)^{r-1} .
\end{aligned}
$$

Letting

$$
g(r)=\frac{1}{r !}\left(1+\left\lfloor\frac{b}{2}\right\rfloor\right)^{r-1}
$$


we have from inequality $(1)$ that $h(r) \leqslant\left(\begin{array}{c}m \\ \lfloor m / 2\rfloor\end{array}\right) \cdot g(r)$ for $1 \leqslant r \leqslant m+1$. Now we maximise $g(r)$. We have

$$
\frac{g(r)}{g(r+1)}=\frac{1+r}{1+\lfloor b / 2\rfloor}= \begin{cases}<1 & \text { if } r<\left\lfloor\frac{b}{2}\right\rfloor \\ =1 & \text { if } r=\left\lfloor\frac{b}{2}\right\rfloor \\ >1 & \text { if } r>\left\lfloor\frac{b}{2}\right\rfloor\end{cases}
$$

We consider two cases:

1. Let $m+1 \leqslant\lfloor b / 2\rfloor$. Then by (2) we have $g(r) \leqslant g(m+1)$ for $1 \leqslant r \leqslant m+1$ and we are done.

2. Let $m+1>\lfloor b / 2\rfloor$. Then by (2) we have $g(r) \leqslant g(\lfloor b / 2\rfloor)$ for $1 \leqslant r \leqslant m+1$ and we are done.

Let now be $m+1>s$. Then we estimate

$$
\max _{r=1, \ldots, s} h(r)
$$

in the same way as above and the result follows.

Let us give some comments on the discrepancy bound from Theorem 1:

1. The ASYMPTOTICS: We discuss the asymptotics of the discrepancy bound in Theorem 1. Assume $\lfloor b / 2\rfloor=\min \{m+1,\lfloor b / 2\rfloor, s\}$ (usually one chooses for $b$ the smallest prime-power larger or equal to $s-1$ ). It is well known that for all $m \geqslant 1$ we have

$$
\left(\begin{array}{c}
m \\
\lfloor m / 2\rfloor
\end{array}\right) \leqslant \sqrt{\frac{2}{\pi}} \frac{2^{m}}{\sqrt{m}}
$$

Then with $N=b^{m}$ we have

$$
\begin{aligned}
D_{N, \gamma}^{*} & \leqslant\left(\begin{array}{c}
m \\
\lfloor m / 2\rfloor
\end{array}\right) \frac{1}{b^{m}} \frac{1}{(1+\lfloor b / 2\rfloor) !}\left(1+\left\lfloor\frac{b}{2}\right\rfloor\right)^{\lfloor b / 2\rfloor} \\
& \leqslant \sqrt{\frac{2}{\pi}} \frac{1}{\sqrt{m}} \frac{2^{m}}{b^{m}} \frac{(1+\lfloor b / 2\rfloor)^{\lfloor b / 2\rfloor}}{(1+\lfloor b / 2\rfloor) !} \\
& \leqslant \frac{\mathrm{e}^{2}}{\pi} \frac{1}{(1+\lfloor b / 2\rfloor)^{3 / 2}} \frac{1}{\sqrt{m}} \frac{(2 \mathrm{e})^{m}}{b^{m}} \\
& =\frac{\mathrm{e}^{2}}{\pi} \frac{1}{(1+\lfloor b / 2\rfloor)^{3 / 2}} \frac{1}{\sqrt{\log _{b}(N)}} \frac{1}{N^{1-\log _{b}(2 \mathrm{e})}}
\end{aligned}
$$

where we used that $n^{n-1} / n ! \leqslant \mathrm{e}^{n} /\left(\sqrt{2 \pi} n^{3 / 2}\right)$ by Stirlings formula and that $\lfloor b / 2\rfloor \leqslant m+1$. Here $\log _{b}$ denotes the logarithm to the base $b$. We summarise: 
COROllary 1. Assume that $\lfloor b / 2\rfloor=\min \{m+1,\lfloor b / 2\rfloor, s\}$. Then for the weighted star discrepancy $D_{N, \gamma}^{*}$ from Theorem 1 we have

$$
D_{N, \gamma}^{*} \leqslant \frac{\mathrm{e}^{2}}{\pi} \frac{1}{(1+\lfloor b / 2\rfloor)^{3 / 2}} \frac{1}{\sqrt{\log _{b}(N)}} \frac{1}{N^{1-\log _{b}(2 \mathrm{e})}}
$$

where $N=b^{m}$ and where e is the Euler number.

2. Consider the problem of integrating a function $f$ in very high dimension $s$, that is, assume that $s$ is huge. As node set for the numerical approximation of the integral we choose a Faure-Niederreiter $(0, m, s)$-net in base $b$. Hence also the base $b$ of the net is huge since a $(0, m, s)$-net in prime-power base $b$ only exists if and only if $b \geqslant s-1$ (see [3]). Now since the number of points of a $(0, m, s)$-net in base $b$ is given by $b^{m}$ we need a moderate $m$ since otherwise our point set is too large and hence the numerical integration by computer is not manageable. In this case the following bound is useful:

Corollary 2. Assume that $m+1=\min \{m+1,\lfloor b / 2\rfloor, s\}$. Then for the weighted star discrepancy $D_{N, \gamma}^{*}$ from Theorem 1 we have

$$
D_{N, \gamma}^{*} \leqslant \frac{\mathrm{e}}{(\lfloor m / 2\rfloor !)^{2}(m+1) 2^{m}}
$$

where $\mathrm{e}$ is the Euler number.

ProOF: From Theorem 1 we get

$$
\begin{aligned}
D_{N, \gamma}^{*} & \leqslant\left(\left\lfloor\frac{m}{m}\right\rfloor\right) \frac{1}{b^{m}} \frac{1}{(m+1) !}\left(1+\frac{b}{2}\right)^{m} \\
& \leqslant \frac{1}{(\lfloor m / 2\rfloor !)^{2}(m+1)} \frac{1}{2^{m}}\left(\frac{2}{b}+1\right)^{m} .
\end{aligned}
$$

Since $m \leqslant b / 2$ we get

$$
\left(\frac{2}{b}+1\right)^{m} \leqslant\left(1+\frac{1}{m}\right)^{m} \leqslant \mathrm{e}
$$

and the result follows.

\section{REFERENCES}

[1] F. Akesson and J.P. Lehoczky, 'Discrete eigenfunction expansion of multi-dimensional Brownian motion and the Ornstein-Uhlenbeck process', (preprint, 2001).

[2] G. Larcher, F. Pillichshammer and K. Scheicher, 'Weighted discrepancy and high-dimensional numerical integration', BIT (2003) (to appear).

[3] H. Niederreiter, Random number generation and quasi-Monte Carlo methods, CBMS-NSF Series in Applied Mathematics 63 (SIAM, Philadelphia, 1992). 
[4] I.H. Sloan and H. Woźniakowski, 'When are quasi-Monte Carlo algorithms efficient for high dimensional integrals?', J. Complexity 14 (1998), 1-33.

Institut für Analysis

Universität Linz

Altenbergerstraße 69

A-4040 Linz

Austria

email: friedrich.pillichshammer@jku.at 